\title{
In Vivo Electrical Conductivity Imaging of Animal Tumor Model at 7T using Electrical Properties Tomography
}

\author{
Prakash Parappurath Vasudevan ${ }^{1,2}$, Reza Aminzadeh ${ }^{2}$, Nele De Geeter ${ }^{3}$, Benedicte Descamps ${ }^{1}$, Luc Dupré ${ }^{3}$, Wout \\ Joseph $^{2}$, Roel Van Holen ${ }^{1}$ \\ ${ }^{1}$ Dept. of Electronics and Information Systems (ELIS), Ghent University, Ghent, Belgium, \\ Prakash.ParappurathVasudevan@UGent.be \\ ${ }^{2}$ Dept. of Information Technology (INTEC), Ghent University, Ghent, Belgium \\ ${ }^{3}$ Dept. of Electrical Energy, Metals, Mechanical Constructions and Systems (EEMMeCS), Ghent University, Ghent, Belgium
}

\begin{abstract}
Ex vivo studies have shown that various diseases alter the electrical properties of tissues compared to healthy nearby tissues. Therefore, electrical conductivity can be used as a diagnostic parameter for e.g. tumor diagnosis. For in vivo measurements, magnetic resonance electrical properties tomography (MREPT) was used and electrical conductivity was reconstructed from the $B 1+$ phase. The technique was first evaluated using homogeneous and heterogeneous phantoms. Then a mouse with a tumor was scanned and the conductivity is reconstructed from the $B 1+$ phase map. The reconstructed conductivity in the phantom experiments was in good agreement with the target conductivity map and the conductivity map of the animal revealed good agreement with the co-axial probe measurement. Our work confirms the possibility of accurate in vivo conductivity assessment in disease.
\end{abstract}

Keywords- Electrical properties tomography; EPT; MRI; conductivity; tumor imaging

\section{INTRODUCTION}

The accurate assessment of dielectric properties has a significant impact on medical applications and diagnosis and is important for radio-frequency (RF) absorption measurements. At present, Gabriel's [1] data base is used as a reference for dielectric properties of human tissues. However, there may be inaccuracies in this database due to ex vivo measurements. Magnetic Resonance Electrical Properties Tomography (MREPT) is a method to assess the electrical properties of tissues in vivo from the RF magnetic field $(\mathrm{B} 1+)$ at the Larmor frequency. This method uses standard magnetic resonance imaging (MRI) equipment without the need of any additional hardware [2-4]. Recent studies have shown the feasibility of phase-based conductivity reconstruction, which uses only the $\mathrm{B} 1+$ phase for the conductivity imaging [5-6].

This study evaluates the feasibility of in vivo conductivity imaging of mouse tumor from the $\mathrm{B} 1+$ phase at $7 \mathrm{~T}$. The feasibility of the method was first evaluated using a phantom and in vivo experiments were conducted on mouse model.

\section{MATERIALS AND METHOD}

Using the homogenous Helmholtz equation for a region with constant conductivity and approximating transmit phase as half of the transceive phase, conductivity was calculated using $[5,6]$ :

$$
\sigma(\mathrm{r})=\frac{\nabla^{2} \varphi_{+}(\mathrm{r})}{\omega \mu_{0}}
$$

where $\varphi_{+}$is the transmit phase, $\omega$ is the Larmor frequency and $\mu_{0}$ is the permeability of free space.

The experimental studies were carried out on a phantom with two different conductivity regions and a mouse with a tumor on the forelimb. The conductivity of the phantom compartments was altered using different concentrations of salt $(\mathrm{NaCl})$. The outer compartment of the phantom is made up of an agar-saline solution (20gr/l Agar, 1.5gr/l CuSO4, $0.05 \mathrm{~mol} / \mathrm{l}$ $\mathrm{NaCl}$ ) with conductivity $0.49 \mathrm{~S} / \mathrm{m}$ and the inner compartment is filled with saline solution $(1.5 \mathrm{gr} / 1 \mathrm{CuSO} 4,0.1 \mathrm{~mol} / 1 \mathrm{NaCl})$ with conductivity $0.96 \mathrm{~S} / \mathrm{m}$. A mouse bearing a prostatic tumor on a forelimb was then used for in vivo studies. The mouse was euthanized after scanning and the tumor conductivity was measured with an Agilent 85070E coaxial probe and a PNA-X network analyzer at $300 \mathrm{MHz}$ for validation of MREPT. All experiments were conducted using a 7T Bruker PharmaScan preclinical MR scanner at Ghent University, Belgium with a quadrature birdcage RF coil with $40 \mathrm{~mm}$ inner diameter. All animal experiments were conducted according to the European guidelines (2010/63/EU) and approved by the local ethical committee of Ghent University.

Two 2D spin echo (SE) images with Repetition Time (TR) of $1600 \mathrm{~ms}$, Echo Time (TE) of $12 \mathrm{~ms}$, resolution of $0.3 \times 0.3 \times 0.9$ $\mathrm{mm}^{3}$ and four averages on five axial slices were used to obtain eddy current corrected B1+ phase images. The total phase of a $\mathrm{SE}$ image [5] is given by (2). 


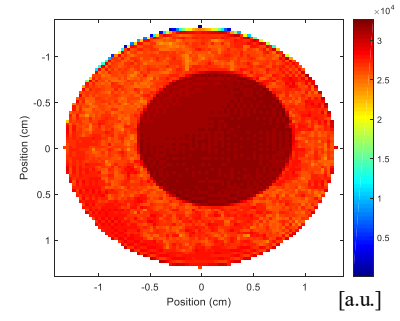

(a)

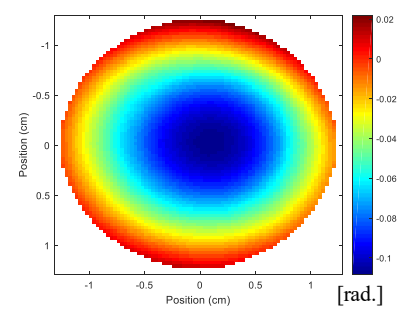

(b)

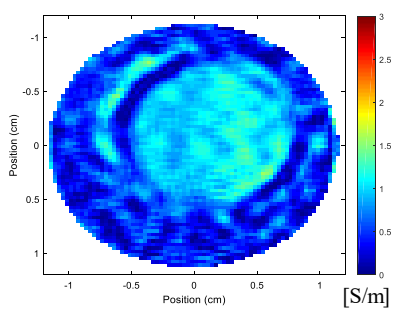

(c)

Fig. 1. Experimental phantom with two different conductivity regions. (a) MR magnitude image (b) B1+ phase image (c) Reconstructed conductivity

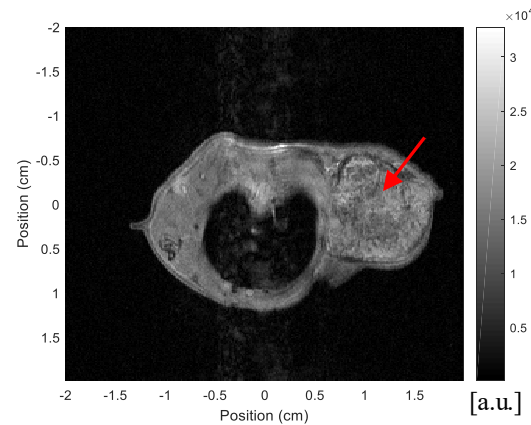

(a)

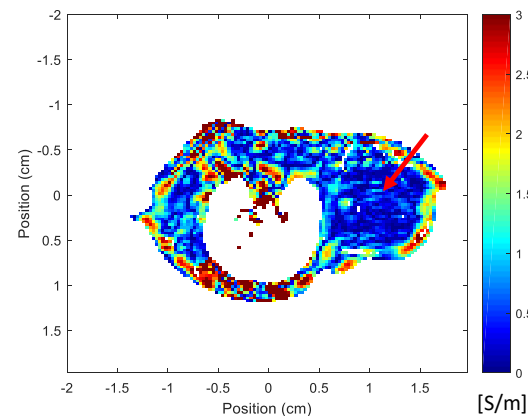

(b)

Fig. 2. In-vivo measurements of animal tumor model (a) T1-weighted image (b) Reconstructed conductivity

$$
\varphi_{s}(\mathrm{r}, \mathrm{TE})=\varphi_{ \pm}(\mathrm{r})+\int_{0}^{\mathrm{TE}} \gamma \mathrm{B}_{\mathrm{e}} \mathrm{dt}
$$

The first term of (2) represents the transceive phase; half of this transceive phase is used as transmit phase for conductivity reconstruction. The second term is the phase contribution from gradient induced eddy currents, which is eliminated using two acquisitions with opposite gradient polarities. The SavitzkyGolay filter is implemented to calculate the Laplacian of the transmit phase in (2).

\section{RESULTS AND DISCUSSION}

Fig. 1 (a), (b) and (c) show the SE magnitude, transmit phase and reconstructed conductivity, respectively at 300 $\mathrm{MHz}$ Larmor frequency. The average reconstructed conductivities for the phantom are $\sigma_{\text {inner }}=0.87 \pm 0.25 \mathrm{~S} / \mathrm{m}$ and $\sigma_{\text {outer }}=0.48 \pm 0.19 \mathrm{~S} / \mathrm{m}$. The results are in good agreement with the expected values of $\sigma_{\text {inner }}=0.96 \mathrm{~S} / \mathrm{m}$ and $\sigma_{\text {outer }}=$ $0.49 \mathrm{~S} / \mathrm{m}$, with relative error of $10 \%$ and $2 \%$ in inner and outer compartments, respectively. Fig. 2 shows the T1-weighted image of the mouse tumor and the reconstructed conductivity image. The average reconstructed conductivity of tumor region was $0.91 \pm 0.65 \mathrm{~S} / \mathrm{m}$. The result was compared with the co-axial probe measurement and relative error was found to be $6 \%$. The reconstructed conductivity image shows artifact around the boundaries due to the approximation in the Helmholtz equation and discrete calculation of the Laplacian.

\section{CONCLUSION}

The results show that the non-invasive conductivity mapping using only the transmit phase is possible in piecewise constant conductivity regions, which confirms the possibility of accurate in vivo conductivity assessment in different pathological conditions. The conductivity determined using this approach can be used for various medical applications e.g. hyperthermia treatment planning. Further experiments will be carried out on living tissues and the effect of signal-to-noise ratio on reconstructed conductivity will be investigated to stabilize the reconstruction algorithm.

\section{ACKNOWLEDGMENT}

The authors thank the financial support of the Research Foundation - Flanders (FWO-V) by grant number G027714N.

\section{REFERENCES}

[1] S. Gabriel, R. W. Lau, and C. Gabriel, "The dielectric properties of biological tissues: II. Measurements in the frequency range $10 \mathrm{~Hz}$ to 20 GHz," Phys. Med. Biol., vol. 41, pp. 2251-2269, Nov. 1996.

[2] Haacke EM, Petropoulos LS, Nilges EW, Wu DH. "Extraction of conductivity and permittivity using magnetic resonance imaging," Phys. Med. Biol., vol. 36, pp. 723-734, 1991.

[3] H. Wen, "Noninvasive quantitative mapping of conductivity and dielectric distributions using RF wave propogation effects in high-field MRI," in Proc. SPIE, 2003, vol. 471-477, p. 5030.

[4] U. Katscher, T. Voigt, C. Findeklee, P. Vernickel, K. Nehrke, and O. Dossel, "Determination of electrical conductivity and local SAR via B1 mapping," IEEE Trans. Med. Imag., vol. 28, no. 9, pp. 1365-1374, Sep. 2009.

[5] T. Voigt, U. Katscher, and O. Doessel, "Quantitative conductivity and permittivity imaging of the human brain using electric properties tomography," Magn. Reson. Med., vol. 66, no. 2, pp. 456-466, 2011.

[6] A. L. van Lier, D. O. Brunner, K. P. Pruessmann, D. W. Klomp, P. R. Luijten, J.J. Lajendijk, and C. A. van den Berg, "B1+ phase mapping at $7 \mathrm{~T}$ and its application for in vivo electrical conductivity mapping," Magn. Reson. Med., vol. 67, no. 2, pp. 555-561, 2012. 